\title{
Retroperitoneal Viscum album extract injection in high drain output patient after renal transplantation
}

\author{
Youngjun Park ${ }^{1}$, Mihyeong Kim², Kangwoong Jun ${ }^{3}$, Jeongkye Hwang ${ }^{2}$, Sangdong Kim ${ }^{4}$, Suncheol Park ${ }^{1}$, Jiil Kim , \\ Sangseob Yoon ${ }^{1}$, Insung Moon ${ }^{6}$

\begin{abstract}
'Division of Transplantation, Department of Surgery, Seoul St. Mary's Hospital, College of Medicine, The Catholic University of Korea, Seoul, Korea ${ }^{2}$ Division of Transplantation, Department of Surgery, Eunpyeong St. Mary's Hospital, College of Medicine, The Catholic University of Korea, Seoul, Korea ${ }^{3}$ Division of Transplantation, Department of Surgery, Bucheon St. Mary's Hospital, College of Medicine, The Catholic University of Korea, Bucheon, Korea ${ }^{4}$ Division of Transplantation, Department of Surgery, Incheon St. Mary's Hospital, College of Medicine, The Catholic University of Korea, Incheon, Korea ${ }^{5}$ Division of Transplantation, Department of Surgery, Uijeongbu St. Mary's Hospital, College of Medicine, The Catholic University of Korea, Uijeongbu, Korea ${ }^{6}$ Division of Transplantation, Department of Surgery, Yeouido St. Mary's Hospital, College of Medicine, The Catholic University of Korea, Seoul, Korea
\end{abstract}

Background: After renal transplantation, high drain output of lymph fluid is one of the causes of delaying postoperative recovery with longer hospital stay. Viscum album extract (sold under the brand name Helixor M) is one of the agents used in pleurodesis procedure when treating malignant pleural effusion, and we evaluated efficacy of this agent in reducing drainage volume and incidence of lymphocele after renal transplantation.

Methods: We retrospectively reviewed medical records of patients with high drain output (>100 $\mathrm{mL}$ at postoperative day [POD] 7) after renal transplantation who undergone Viscum album extract injection via drain $(n=23)$ or conservative care $(n=23)$. The primary end point is reduced volume in drain output at POD 14 from POD 7. The secondary end point was postoperative recovery as reflected by the duration of hospitalization and incidence of lymphocele during 3 months after operation.

Results: Reduction in drain output at POD 14 from POD 7 was larger in the Viscum album extract injection group than in the conservative care group $(226.5 \mathrm{~mL}$ vs. $83.3 \mathrm{~mL} ; \mathrm{P}=0.010)$. Duration of hospitalization after operation was shorter in the Viscum album extract injection group than in the conservative care group (16.0 days vs. 19.5 days; $P=0.012$ ). Ratio of patients who discharge with drain kept was also less in the Viscum album extract injection group than in the conservative care group (17.4\% vs. $52.2 \% ; P=0.029$ ).

Conclusions: In this study, reduction in drain output at POD 14 from POD 7 was larger and duration of hospitalization was shorter after renal transplantation when Viscum album extract was injected via drain, suggesting that Viscum album extract injection could be considered for faster postoperative recovery in high drain output patients after renal transplantation.

Corresponding author: Youngjun Park

E-mail: cmc201133035@gmail.com

(c) The Korean Society for Transplantation

This is an Open Access article distributed under the terms of the Creative Commons Attribution Non-Commercial License (http://creativecommons.org/licenses/by-nc/4.0/) which permits unrestricted non-commercial use, distribution, and reproduction in any medium, provided the original work is properly cited. 